\title{
Detecting and Tracking Breeding Cows from Bird's Eye Video of Pasture
}

\author{
Ryo Nishide ${ }^{\mathrm{a},{ }^{*},}$ Yoji Hosomi ${ }^{\mathrm{a}}$, Takenao Ohkawa ${ }^{\mathrm{a}}$, Kenji Oyama ${ }^{\mathrm{b}}$, Chikara Ohta $^{\mathrm{c}}$ \\ ${ }^{a}$ Graduate School of System Informatics, Kobe University, Kobe 657-8501, JAPAN \\ ${ }^{\mathrm{b} F o o d}$ Resources Education and Research Center, Kobe University, Kasai 675-2103, JAPAN \\ ${ }^{c}$ Graduate School of Science, Technology and Innovation, Kobe University, Kobe 657-8501, JAPAN \\ *Corresponding Author: nishide@ port.kobe-u.ac.jp
}

\begin{abstract}
Breeding cows are known to engage in social activities, such as interacting with other cows and forming groups similarly to humans. This paper proposes a method to extract information of interaction between cows, regarding it as a primary element of sociality. In order to extract such information, we record bird's eye pictures in a pasture by a fixed point camera set on a silo and analyze the interaction from the video data. Then, the movements of cows are tracked from bird's eye video data to detect interaction. As the cows have little features on image, background subtraction method is employed without modeling the target object. The effectiveness of the proposed method has been examined in experiments with the recorded bird's eye pictures. Our ultimate goal is to recognize the health conditions and stress of cows through their mutual interactions.
\end{abstract}

Keywords: image processing, cow, interaction, location tracking, social behavior.

\section{Introduction}

The advancements of computer technologies have paved way to store and analyze big data in recent years. These data are used for data mining in various fields in order to acquire valuable scientific knowledge. Agriculture field has also changed from experience-based method to scientific knowledge-based method to improve productivity. We focus on livestock field, which biological data (bovine growth and body condition data) and work data (amount of feed and medicine information) are collected from individual cow, and used to improve feeding skills and management efficiency.

Most existing methods collect and analyze information of individual livestock, and not much work has been done on the sociality of livestock raised in groups. Cows (which we focus on) tend to engage in social activities similarly to human, such as mutual interaction and forming groups $^{(1)}$. In this study, we focus on the interaction through physical contact such as affiliative behavior and mounting. Our goal is to predict the state of cows by extracting the interaction, which cannot be analyzed from methods targeted on individual cow. In this paper, the bird's eye image in a pasture is recorded, and the method to automatically extract and track the cows in the image is proposed.

Several methods to detect a certain object from video data captured by fixed point camera have been presented and developed ${ }^{(2,3)}$. Compared to other existing methods which mainly target on detecting human, our work on cows do not have as much image features as they have on humans, and therefore, the same method cannot be applied to our work. Cows are extremely difficult to detect due to their monotonous color and slow movement. Focusing on the characteristic that large amount of data exist where cows do not appear on a bird's eye video, we apply background subtraction method ${ }^{(4)}$ to extract cows.

In order to show efficiency of our method, we have conducted tracking experiment of cows. The tracking accuracy has been evaluated by F-measure, and then our method was compared with the method using fixed background image. The experiment has revealed that our method which serially selected the background image with background subtraction method for each input image has contributed to improving the tracking accuracy of cows.

\section{Overview of Extracting Bird's Eye Video}

\subsection{Characteristics of Cows and Their Environments}

There are about forty Japanese Black breeding cows raised at the Food Resources Education and Research Cen- 


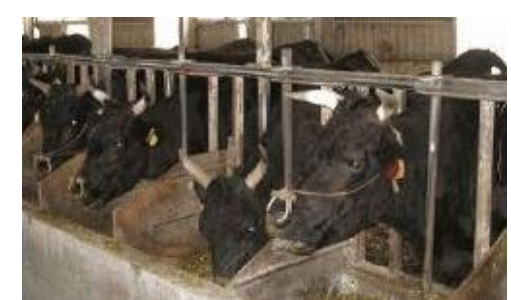

Fig. 1. Breeding cows in a cowshed.
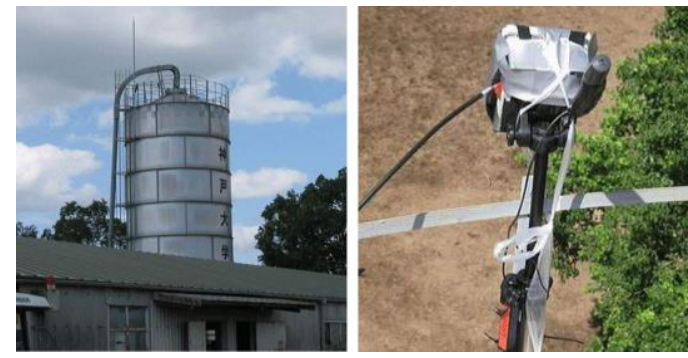

Fig. 2. A silo for setting a camera.

ter at Kobe University as shown in Fig. 1. Breeding cows are raised as economical animals which efficiently increase their numbers by giving birth. Heifers are raised as breeding cows, and steers are fed up as fattening cows for human consumption. Generally, breeding cows and milk cows are raised as a group, and they are known to have sociality because they form groups and interact with each other within groups similarly to humans ${ }^{(1)}$.

The width of a pasture is 1.4 ha, and cows can freely walk around the pasture and the cowshed. They are fed in the cowshed at the same time of day, but they also eat grasses naturally grown in the pasture. They can drink water either at the cowshed or at the drinking trough in the pasture.

\subsection{Obtaining Bird's Eye Video}

Cows spend most of the time in the pasture. Thus, we consider that their social activity on daytime can be grasped by observing their movement and behavior in the pasture. In this study, a camera is installed on the roof of the silo (a structure to store food for cows) near the pasture as shown in Fig. 2, and it records the cow's activity in the pasture on a bird's eye view. The devices used for recording the pasture are as follows:

- Network Camera (SNC-XM637)

- $\quad$ Laptop Computer (Windows Vista)

- $\quad$ Portable Hard Disk (HDE-U2.0J)

- $\quad$ HUB with PoE (BSL-PoE-G2105U)

- $\quad$ LAN Cable (30m)

- $\quad$ USB Cable

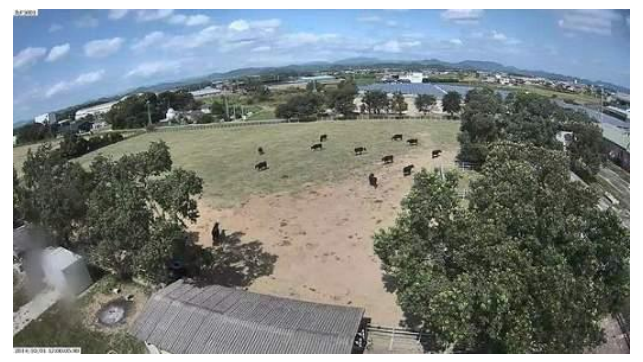

Fig. 3. Bird's eye photo.

The specification for the network camera is approximately 2,140,000 pixels, fixed-focus lens, $113^{\circ}$ horizontal angle of view, and full high-vision with a resolution of $1,920 \times 1,080$. The camera is installed with fish-eye lens though the lens can distort the actual bird's eye video data.

The power is supplied to the camera on a silo using a HUB enabled with PoE electric supply, and the recorded video data is sent through the network and stored in the server. The recording time is set from $6 \mathrm{am}$ to $6 \mathrm{pm}$ as a certain brightness is required to record the pasture. Recording started from September 27, 2014 and is still on-going. Fig. 3 shows a snapshot of the obtained bird's eye video. We aim to verify the kind of social activity the cows perform in the pasture, and whether the interaction between cows exists by analyzing the bird's eye video data.

\subsection{Interaction of Cows}

There are various social activities of cows ${ }^{(1,5)}$. For example, smaller groups (denoted as grouping) are formed depending on a hierarchy of the group, and they spend much time in a group. When a new cow joins the group, other cows show hostile behavior and they bunt each other to decide the hierarchy in a group. We focus, in this study, on a mutual interaction by physical contact of cows rather than grouping. Physical contact has possibilities to identify relations of cows from friendly or hostile behavior, cow's estrus from mounting or standing, which may be a clue for understanding the sociality to identify the conditions or behavior in their community.

\section{Pre-processes for Extracting Cows}

\subsection{Calibration of Distorted Bird's Eye Video Data}

As a preliminary process to extract cows, it is necessary to calibrate the bird's eye video data because the fish-eye lens uniquely distort the image data. Image can be undistorted based on the pin-hole camera model ${ }^{(6,7)}$. Pin-hole camera is a camera with a small hole in the aperture, and the image is captured through the aperture. In 


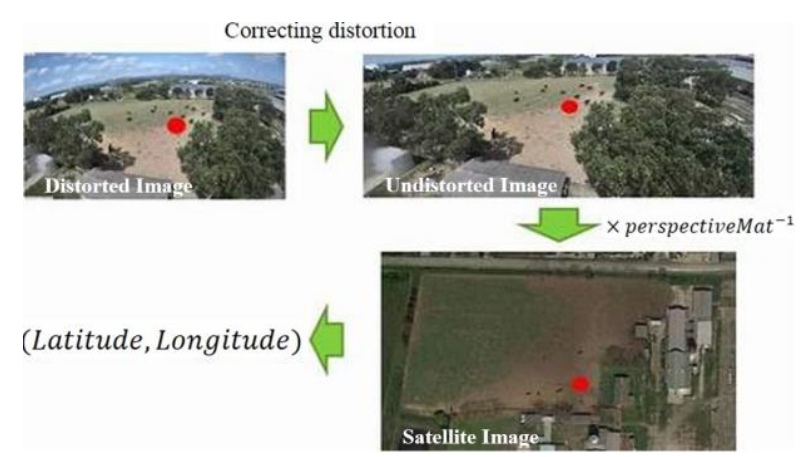

Fig. 4. Procedure to calculate latitude and longitude.

order to calibrate, it is necessary to calculate the internal parameter (focal distance, optical center) of the camera. In this work, we applied method using chessboard pattern ${ }^{(8)}$ to calculate the internal parameter. Our chessboard pattern consists of 11 horizontal and 8 vertical cells, each with the size of $2.4 \mathrm{~cm} \times 2.4 \mathrm{~cm}$.

Fig. 4 shows an undistorted image compared with the distorted image. The bird's eye video is calibrated appropriately as the fence around the pasture is straightly connected. The image sizes are different because a certain part of area shot with fish-eye lens is lost due to calibration.

\subsection{Estimation of Cow's Size and Location}

\section{[Location Estimation of Cows]}

To estimate the size of cows from image, it is necessary to calculate the distance from the camera to the cows accurately. We consider using the latitude and longitude of cow's location in the undistorted image. If the latitude and longitude is obtained, it is rather simple to calculate accurately by comparing the satellite photo and bird's eye photo of the pasture.

Projective transformation ${ }^{(9)}$ is used to compare the location on satellite photo and undistorted bird's eye photo. It enables the latitude and longitude of satellite photo to correspond with the same location in the bird's eye photo. The projective transformation matrix (perspectiveMat in Fig. 4) can be obtained by corresponding the coordinates of four corners of pasture on a satellite photo and bird's eye photo respectively. Cow's latitude and longitude can be determined by calculating the corresponding coordinates of satellite photo to the bird's eye photo using the inverse of projective transformation matrix. The entire procedure is illustrated in Fig. 4. The red spot is the corresponding spot to each of the photo.

\section{[Size Estimation of Cows]}

Equation (1) calculates the distance $d$ between camera and cow using the latitude and longitude of cow and camera discovered beforehand ${ }^{(10)}$.

$$
d=\sqrt{\left(y-y_{\text {camera }}\right)^{2} M^{2}+\left(x-x_{\text {camera }}\right)^{2} N^{2} \cos ^{2} \mu_{y}}
$$

Note that $(x, y)$ is the cow's latitude and longitude, ( $\left.x_{\text {camera, }} y_{\text {camera }}\right)$ is the camera's latitude and longitude, $M$ and $N$ are the Meridian radius of curvature and Prime vertical radius of curvature, and $\mu_{y}$ is the arithmetic mean of cow and camera's latitude.

Cows in a bird's eye video appear large when they are close to the camera, and appear small and sometimes difficult to see with our naked eyes when they are far from the camera. In other words, the cow's size becomes proportionally small in respect to the distance of the camera and cow. Formula to find the distance between cow and camera, and the size of cow can be obtained by performing linear regression $^{(11)}$ using the size of cow as objective variable and the distance as explanatory variable. However, even if the cows stay at a same location, the size of cow differs according to its facing direction. Therefore, the postures of cows are classified into two types, namely horizontal and vertical direction, and the regression must be performed separately. In order to classify the horizontal and vertical posture of cows, the Height and Width are compared, and if Width < Height then it is vertical direction, otherwise if Width $\geq$ Height then it is horizontal direction.

Equation (2) can be obtained using the height and width of cow as objective variables to perform linear regression.

$$
\left\{\begin{array}{l}
\text { width }_{\mathrm{est}}=a_{w} d+b_{w} \\
\text { height }_{\mathrm{est}}=a_{h} d+b_{h}
\end{array}\right.
$$

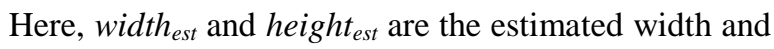
height of a cow, $a_{w}, a_{h}, b_{w}, b_{h}$ are the coefficients obtained by regression.

\section{Detection of Cows}

\subsection{Background Subtraction Method}

Background subtraction method is often used to detect animals in the video recorded from fixed camera. Differential image $I_{\text {diff }}$ is generated from background only image $I_{\text {back }}$ and input image with target object $I_{\text {input }}$, and thresholding is performed on differential image in order to detect the area with the target object.

$$
I_{\text {diff }}=\left|I_{\text {input }}-I_{\text {back }}\right|
$$

In this study, the bird's eye video data is obtained from fixed camera. We consider that cows in the picture can be detected by using the picture without cows as the reference image. However, the background of reference image and 
input image must be the same, and difference of brightness or shaking objects by the wind can cause misdetection. Several methods have been developed to deal with these problems, such as a method to update the background model with the newly obtained image accordingly to deal with slight changes of brightness ${ }^{(3,12)}$. However, it is not applicable to our work as the color of cows are monotonous and also as the cows do not move frequently, which may identify them as parts of the background or noises.

Some other works employ particle filters ${ }^{(2,13)}$ in order to extract the area with target objects by calculating the probability of the target existing in each pixel. In our work, as the colors of cows are similar to the color of ground, misdetection is likely to occur. Methods using local feature $^{(14)}$ also have problems related to the color of cows, as the feature points of cows are frequently matched with the corresponding feature points of the background. Our method focuses on a property that many background images without cows can be obtained, and then extends the basic background subtraction method in order to detect the cows.

In our collected video data, many pasture image data without cows can be easily obtained when they are being fed at the cowshed. Background image data with various seasons and time is stored in the database. We aim to improve the detection accuracy of cows with background subtraction method by selecting the optimal reference image depending on the input image data of cows.

\subsection{Background Subtraction to Extract Cow's Region}

\section{[Creation and Search of Background Image Database]}

The brightness of the reference images must be similar with the input images because noise tends to occur at regions other than cows. When creating the database, outdated video data may be inappropriate for the selection because the brightness might have changed. Therefore the data is limited to $L$ days prior, and image without cows is selected to store to the database. Cows are gathered in the cowshed from around $9 \mathrm{am}$ to $12 \mathrm{pm}$, and there are no cows in the pasture. The video data recorded during this time has a similar brightness environment, and can be used as a background image.

However, if the images stored in the database are limited to this time periods, it will be difficult to extract the cows unless the brightness is the same with the stored images. Thus, it searches the data captured with the same time $T$ the input image was captured, because it can be considered that the brightness environment recorded at same time of day is similar. The images recorded between $T-W$ to

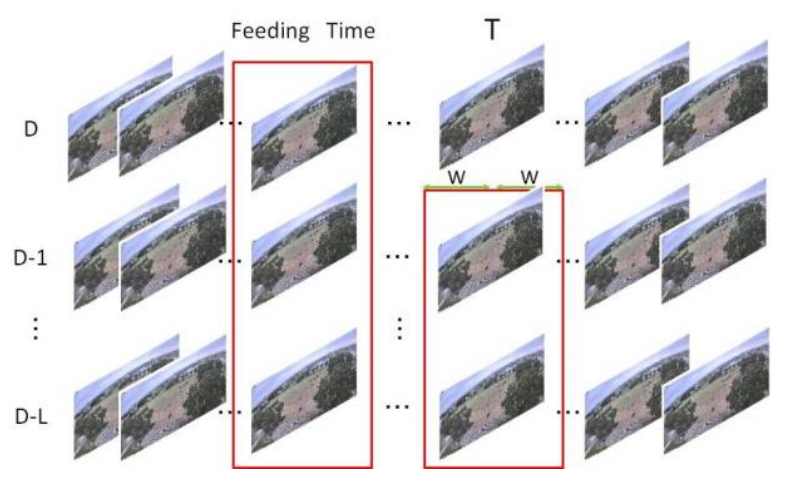

Fig. 5. Searching range for creating reference images.

$T+W$ are searched because it is necessary to select images with a similar brightness environment. Image groups during the feeding time definitely have no cows in the image, so if it matches to the brightness condition then it is appropriate as a reference image, and image groups during this time will be the search candidates disregarding the recorded time. Fig. 5 illustrates the entire search range with red square.

\section{[Extraction of Cow's Region]}

Background image database is created and searched in order to obtain reference images. Desirable background image for reference image can be obtained by completely eliminating the region without cows. To do so, we create differential image $I_{\text {diff }}$ from Equation (3), and then generate binary image from threshold process in Equation (4).

$$
I_{t h}(x, y)= \begin{cases}1 & I_{\text {diff }}(x, y) \geq \text { threshold } \\ 0 & I_{\text {diff }}(x, y)<\text { threshold }\end{cases}
$$

$I_{t h}$ is a binary image generated from threshold process, and $(x, y)$ are the coordinates on the image. threshold is the average luminance value of the whole pixel which the value is not 0 .

In this way, binary image $I_{t h}$ with minimum number of pixel which does not have the value 0 can be obtained as a reference image. However, if the one and only image is selected from background image database, misdetection is likely to occur from noises caused by trees swaying, or even the images with cows can be chosen as background image.

The binary image $I_{t h}$ obtained from input image and each image in a search range with pixels that the value is not 0 are assigned as evaluation value, and all of the images in the search range is ranked (with smaller values as higher ranks). Then, using the background images with top $K$ ranked evaluation value, we believe that cow's region is extracted with high precision while removing the noise.

When selecting top $K$ background images photographed in a close time span, the brightness tends to be 


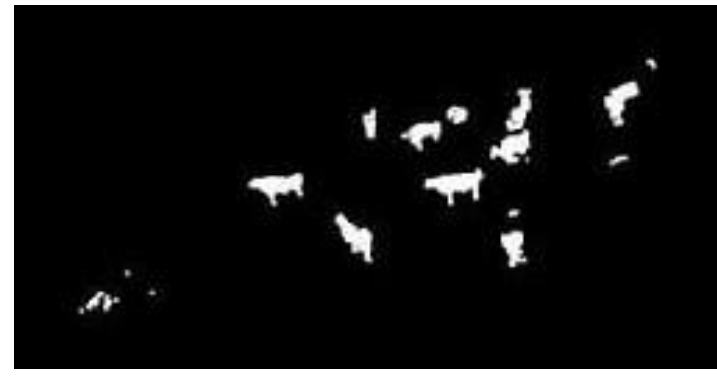

Fig. 6. Extracted cow's region from input image.

similar. Therefore, the image with highest evaluation value is selected first, then the next image is chosen from the ones that are captured from time $W_{T}$ prior or later to the image selected first. Furthermore, the following image is chosen from the ones that are captured from time $W_{T}$ prior or later to all of the previously selected images. This is performed recursively to avoid selecting background images with similar brightness.

Let $I_{t h}^{1}, \ldots, I_{t h}^{K}$ be a group of selected $K$ binary background images. The opening and closing of morphology transformation is applied for each binary image to eliminate more noise ${ }^{(15)}$. Opening process removes small noise such as swaying trees and deformation of ground on which the cows have stepped over. Closing process removes the noise inside the cow's region and smoothen the contour, which enables to easily extract the cow's region in the image.

Flag matrix $I_{\text {detect }}$ in Formula (5) is used to extract the cow's region.

$$
I_{\text {decect }}(x, y)=\prod_{i=1}^{K} I_{t h}^{i}(x, y)
$$

Flag matrix is expressed from multiple of $K$ binary image groups where each background image is assigned as 1 if it is a cow's region, and 0 if it is not. Therefore, when inputting images, if there are no foreground object in all background images, then cow's region for each flag matrix will not be extracted.

\section{[Detecting Cows]}

Fig. 6 shows an example of binary image of cow's region extracted by our method. In order to find the center and size of each cow's region in binary image, bounded rectangle is extracted for each region. In a bounded rectangle, a few noise remains because flag matrix cannot completely eliminate it. Most of the noise is caused by deformation of ground due to the cow's footprint or dung, and their size is also proportional to the distance from camera. However, the noise is relatively small compared to cows in the same location. Therefore, latitude, longitude and the size of cows can be calculated from the method in Section
3.2 and the height and width of a bounded rectangle are compared in order to remove the noise.

To remove the noise, the images are classified according to the direction of cows, and the estimated values are calculated using Equation (4) for each horizontal and vertical direction in the same way as Section 3.2. The estimated value and its height or width is compared and then identified as either noise or cow. During this process, the measured value sparsely variates according to the estimated value, and tends to identify the bounded rectangular region of actual cows as noises. Thus, the lower and upper confidence interval of $95 \%$ is used instead of using the estimated value itself.

However, physical contact between cows may cause two cows to exist in one region. In such cases, the region of cows may expand. Setting the upper limit may fail to detect the cows, so only the lower limit is used in such cases. Therefore, if the height and width of cows obtained from the linear regression is over $95 \%$ of the confidence interval then it is recognized as cow, otherwise it is processed as noise.

\subsection{Tracking Cows}

Tracking cows are enabled by matching the bounded rectangle in time $T$ and $T-1$ respectively as the same cow. Cow's moving speed is extremely slow, thus the location of cow between time $T$ and $T-1$ does not change drastically. Therefore, the central coordinates of bounded rectangle in time $T$ and the minimum distance of rectangle (in time $T-1)$ are matched, and these processes are performed recursively in order to track the cows as shown in Equation (6).

$$
\left\{\begin{array}{l}
x^{*}=x_{T-1}+v_{T-1} \\
v_{T}=\alpha\left(x_{T}-x_{T-1}\right)+(1-\alpha) v_{T-1}
\end{array}\right.
$$

Note that $x^{*}$ is the estimated location of cow in time $T$, $x_{T}$ is the central coordinates of cow's bounded rectangular region in time $T$, and $v_{T}$ is the cow's velocity in time $T$. In the bottom equation, $\alpha$ is the velocity in time $T$, which is the weighted average of the amount of location change from $T-1$ to $T$ and velocity of time $T-1$, and the value is the real number between 0 to 1 .

If the region expected to be cow is matched based on the length between the central coordinates, it may address the nearby noise as a cow by mistake as stated previously. Here, we focus on the size of cow and noise, and the rectangles are matched with the ones that have the minimum summation of the length of center of bounded rectangle and 
four corresponding corners of the bounded rectangle. This method can match based on location and size, and has possibilities to reduce misdetection of the target to track. These processes can be expressed with Equation (7).

$$
r e c_{i}^{T-1} \rightarrow \min _{r e c_{j}^{T}}\left(\sum_{k=0}^{3} d\left(C_{r e c_{j}^{T}}[k], C_{r e c_{j}^{T}}^{*}[k]\right)+d\left(x^{*}, \operatorname{ctr}\left(r e c_{j}^{T}\right)\right)\right)
$$

Let $\operatorname{rec}_{i}^{T}$ be the $i$-th bounded rectangle in time $T$. $C_{r e c_{i}^{T}}[k]$ represents four corners of bounded rectangle $\operatorname{rec}_{i}^{T}$. $k=0$ is the upper left, $k=1$ is the upper right, $k=2$ is the bottom right, $k=3$ is the bottom left corner of the rectangle. $C_{r e c_{i}^{[}}^{*}{ }^{[k]}$ is the rectangle in time $T-1$, which the four corners of time $T$ are expected to be at. Each corner can be estimated with Equation (8).

$$
C_{\operatorname{rec}_{i}^{T}}^{*}[k]=C_{r e c_{i}^{T-1}}[k]+v_{T-1}
$$

$D\left(X_{1}, X_{2}\right)$ is the Euclidean Distance of point $X_{1}$ and $X_{2}$. $x^{*}$ expresses the central location of a cow from time $T-1$ to the estimated central location in time $T$ using Equation (6), where $\operatorname{ctr}\left(\operatorname{rec}_{i}^{T}\right)$ is the central coordinates of rectangle $\operatorname{rec}_{i}^{T}$. If there is a large noise similar to the size of nearby cows, it may not be able to match with the cows correctly. To avoid this problem, assuming that cows do not have a similar color with trees or grasses even though the color of ground is similar, color model can be generated based on RGB value of cow's bounded rectangular area in time $T-1$, and thresholding is processed based on similarity degree. In detail, to a bounded rectangle in time $T$ matched with a rectangle in time $T-1$, in order to input a RGB value of whole pixels in a rectangle, average likelihood of the color model generated from a rectangle in time $T-1$ is calculated. If the average is below a certain threshold $\lambda$, then the rectangle is determined as a misdetection as a noise, and performs rematching. This matching is performed recursively until the rectangle exceeding the threshold is found, and the misdetection of region which is clearly different from cows (such as trees, grasses, etc.) can be avoided.

\section{Experiment}

\subsection{Dataset and Parameter Settings}

The bird's eye video data taken from a silo nearby the pasture on October 1-3, 2014 was used to construct a background image database, and the video data taken on October 3, 2014 was used as a test data. Thus, $L$ in Section 4.2 is assigned as 2 . These video data was sampled every second, and image data was extracted for experiment. The average speed of cows are very slow and it does not affect much on the experiment results even if it is roughly sampled to some
Table 1. Number of images and feeding time.

\begin{tabular}{|c|c|c|}
\hline Date & Feeding Time & Num. Images \\
\hline Oct.1 & $9: 12: 03-11: 56: 00$ & 9,837 \\
\hline Oct.2 & $9: 17: 54-11: 47: 06$ & 8,952 \\
\hline Oct.3 & $9: 23: 09-11: 27: 36$ & 7,467 \\
\hline
\end{tabular}

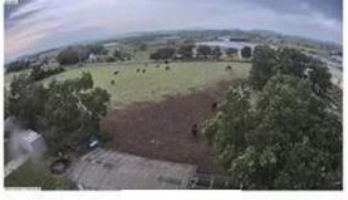

$17: 05: 50$

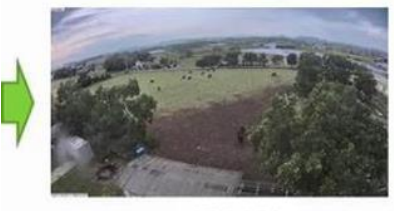

$17: 06: 30$

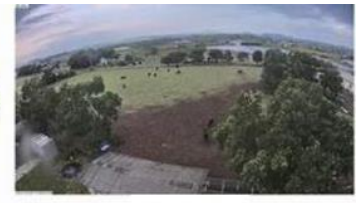

17:06:00

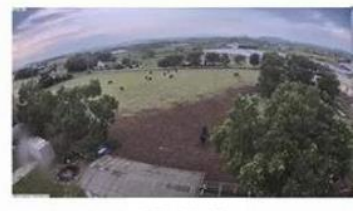

17:07:00
Fig. 7. Dataset collected from 17:05:50 to 17:07:00.

extent. Furthermore, even if this experiment was conducted with only part of the video data, it would be necessary to reduce the running time as a huge amount of data was processed. Tab. 1 shows the feeding time and number of images used to construct the database. Dataset was generated on October 3, 17:05:50 - 17:07:00 (for 70 seconds), and 70 images as shown in Fig. 7 were obtained.

Approximately 10 cows appeared in the dataset. In the dataset, the cows separate at either the exposed ground on the foreside or the grassy field on the backside. We have assumed that the appropriate time of day for experiment is the time when we were able to observe interactions between cows, considering that our long-term objective is to automatically detect interactions between cows. Moreover, images taken during feeding time in Tab. 1 and input images taken 30 minutes prior and later are used to construct the background image database. If $W$ is 1,800 [sec], then the background image database has $9,837+8,952+7,467+$ $1,800 \cdot 2=29,856$ images.

Three patterns of experiment were performed generating flag matrix with the images ranked top, top third and top fifth $(K=1,3,5)$. In addition, it is necessary to mix the choices with different brightness when choosing high ranked background images, and therefore images over $W_{T}=$ 120 [sec] apart were selected. The parameter for updating the velocity was set as $\alpha=0.8$. Finally, the parameter used for thresholding when calculating average likelihood of bounded rectangle extracted from color model of cows was set as $\lambda=0.1$. 
Table 2. Estimated cow's height and width.

\begin{tabular}{|c|c|}
\hline Camera Dist. & Height \\
\hline 2001.9 & 90 \\
\hline 2758.6 & 75 \\
\hline 3466.6 & 63 \\
\hline 3267.6 & 49 \\
\hline 3554.8 & 46 \\
\hline 3634.3 & 39 \\
\hline 5398.0 & 28 \\
\hline 3694.6 & 24 \\
\hline 5274.8 & 21 \\
\hline 6383.0 & 15 \\
\hline
\end{tabular}

\begin{tabular}{|c|c|}
\hline Camera Dist. & Width \\
\hline 2994.1 & 111 \\
\hline 3230.2 & 90 \\
\hline 2945.4 & 83 \\
\hline 4146.3 & 73 \\
\hline 3426.7 & 58 \\
\hline 3513.5 & 44 \\
\hline 4773.2 & 32 \\
\hline 5165.3 & 31 \\
\hline 6010.3 & 24 \\
\hline 6383.0 & 16 \\
\hline
\end{tabular}

Table 3. Parameter of result in regression

\begin{tabular}{|c|c|}
\hline \multicolumn{1}{c}{ Height } \\
\hline$a_{h}$ & $-0.01608 \pm 0.0327$ \\
\hline$b_{h}$ & $108.4109 \pm 13.54315$ \\
\hline$P\left(a_{h}\right)$ & 0.001168 \\
\hline$P\left(b_{h}\right)$ & 0.0000435 \\
\hline
\end{tabular}

\begin{tabular}{|c|c|}
\hline$a_{w}$ & $-0.02177 \pm 0.004575$ \\
\hline$b_{w}$ & $149.0156 \pm 20.12905$ \\
\hline$P\left(a_{w}\right)$ & 0.00143 \\
\hline$P\left(b_{w}\right)$ & 0.000079 \\
\hline
\end{tabular}
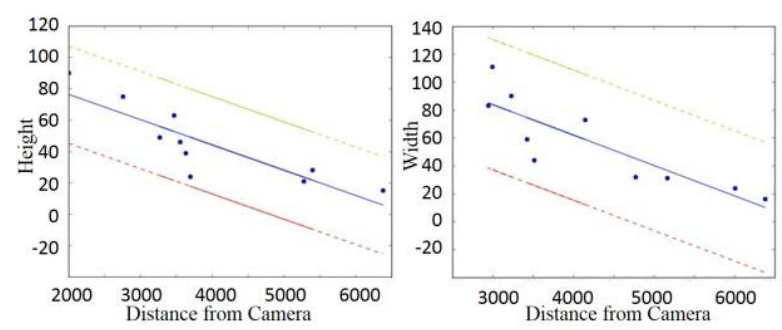

Fig. 8. Regression line.

\subsection{Experiment of Cow Size Estimation}

Linear regression was performed with distance $d$ of cow to the camera and estimated height est $_{\text {and }}$ width ${ }_{\text {est }}$ of cow using data in Tab. 2. The height, width and central coordinates of a cow were collected through manual measurements, and the distance to the cow was extracted with Equation (1). The height and width parameter obtained from linear regression in Equation (2) and $P$ value is shown in Tab. 3. The confidence interval is $95 \%$.

The graph of height and width obtained from regression is shown in Fig. 8. The straight blue line is the regression line, dotted red line is the minimum confidence interval $95 \%$, and dotted yellow line is the maximum confidence interval $95 \%$. Only the minimum confidence level is used for detecting the cows.

\subsection{Experiment of Tracking Cows}

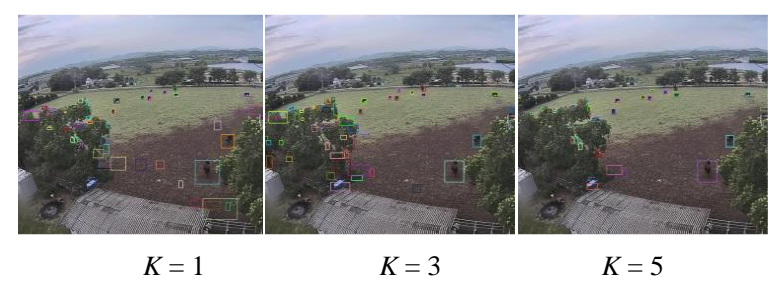

Fig. 9. Detection result with colored rectangles.

Table 4. Comparison of F-measure w.r.t. $K$ value.

\begin{tabular}{|c|c|c|}
\hline$K$ & F-measure (proposed) & F-measure (comparison) \\
\hline 1 & 0.286217 & 0.255158 \\
\hline 3 & 0.442992 & 0.321314 \\
\hline 5 & 0.515692 & 0.495620 \\
\hline Average & 0.414967 & 0.357364 \\
\hline
\end{tabular}

An experiment was conducted to select an appropriate background image for each input image using the dataset.

For comparison, the proposed method was applied only to the first input image, constructed the database and selected an appropriate background image group. The rest of them kept on using the same background image group. F-measure, which is defined as a harmonic means of precision and recall is applied to the evaluation. If there is a central coordinate of a cow manually allocated inside the bounded rectangle, then it is considered that a cow is being tracked correctly. In case multiple cows are in a single rectangle, they are also considered to be correctly detected.

Fig. 9 shows the results of proposed method with different $K$ values for the dataset. It can be observed that $K=1$ and $K=3$ has lots of noises, but the noises are greatly reduced when $K=5$.

\subsection{Multiple vs. Single Background Image(s)}

Tab. 4 validates that F-measure for proposed method using multiple background images is significant in comparison to the method using only a single background image. It can be considered that the noise was effectively reduced in proposed method. Furthermore, the characteristics of proposed method to select an optimal background image for each input image is effective for detecting and tracking cows. Moreover, F-measure increases with the increase of $K$ value, and the proposed method outperforms comparison method disregarding of its increase of $K$ value. These results indicate that it is efficient to detect cows totally from multiple background images rather than from a single image. The noise remaining in a single background subtraction image may be removed in other background subtraction images. 


\section{Conclusion}

We have proposed method to detect and track cows by a bird's eye video of pasture. Background image database is constructed based on input images, and the images are stored and ranked. Then, optimal background image is chosen recursively to provide variety of images from different time and brightness, and the detection accuracy of cows has been refined using background subtraction method. Our method of using multiple background images was compared with method using a single fixed image, and the efficiency of our method was examined.

While focusing on the sociality of cows, our ultimate goal is to develop a system to detect health condition, estrus, and stress of cows by detecting the interaction between cows from bird's eye video. Automatic detection and tracking of cows proposed in our method are needed for detecting interaction between cows, thus we believe that this work can contribute significantly to our goal.

For future works, it is necessary to deal with detecting cows in a blind spot of camera by employing sensor data to obtain location of cows, or distinguishing the cows from their own shadows or the shadows of other objects. There are some other issues that the cows cannot be detected, so recursive background subtraction method to enhance the detection accuracy of cows must also be considered. An efficient method to generate various brightness images will be required to deal with different weather or sunshine. Lastly, the automatically tracking method of breeding cows proposed in this paper can be combined with the auto classification of interactions generated among breeding cows, applying Bag-of-Visual-Words ${ }^{(16)}$. Moreover, the method of detecting interaction of breeding cows by computing from bird's eye video data needs to be further explored.

\section{Acknowledgment}

This work was supported by JST CREST Grant Number JPMJCR1682 and JSPS KAKENHI Grant Number 15K12099 (Grant-in-Aid for Challenging Exploratory Research).

\section{References}

(1) D. P. Dickson, G. R. Barr and D. A. Wieckert : "Social relationship of dairy cows in a feed lot", Behaviour, Vol. 29, No. 2, pp. 195-203, 1967

(2) J. Czyz, B. Ristic and B. Macq : “A particle filter for joint detection and tracking of color objects", Image and Vision Computing, Vol. 25, pp. 1271-1281, 2007

(3) Z. Zivkovic : "Improved adaptive gaussian model for background subtraction", ICPR, Vol. 2, pp. 28-31, 2004

(4) M. Piccardi : "Background subtraction techniques: a review", IEEE International Conference on Systems, Man and Cybernetics, Vol. 4, pp. 3099-3104, 2004

(5) K. Millera and D. G. M. Wood-Gush : "Some effects of housing on the social behaviour of dairy cows", Animal Production, Vol. 53, No. 3, pp. 271-278, 1991

(6) G. Bradski and A. Kaehler : "Learning openCV: computer vision with the openCV library", Oreilly \& Associates Inc., 2008

(7) J. Heikkila and O. Silven : "A four-step camera calibration procedure with implicit image correction", IEEE International Conference on Computer Vision and Pattern Recognition, pp. 1106-1112, 1997.

(8) Z. Zhang : "A flexible new technique for camera calibration", IEEE Transactions on Pattern Analysis and Machine Intelligence, Vol. 22, No. 11, pp. 1330-1334, 2000

(9) W. R. Bion : “Transformations”, Karmac Books, 1984

(10) K. Hubeny : “Weiterentwicklung der gauss'schen mittelbreitenformeln", Zeitschrift Vermess, Vol. 84, pp. 159-163, 1959

(11) P. L. Rosin : "Further five-Point fit ellipse fitting", Graphical Models and Image Processing, Vol. 61, No. 5, pp. 245-259, 1999

(12) P. Kaewtrakulpong and R. Bowden : “An improved adaptive background mixture model for realtime tracking with shadow detection", In Proc. 2nd European Workshop on Advanced Video Based Surveillance System, Vol. 5308, pp. 135-144, 2001

(13) S. S. Sakharkar, S. D. Kamble and A. S. Khobragade : "Object detection and tracking using particle filter", IJCTT, Vol. 22, No. 1, pp. 16-19, 2015

(14) P. F. Alcantarilla, J. N. and A. Bartoli : "Fast explicit diffusion for accelerated features in nonlinear scale spaces", BMVC, Vol. 13, pp. 1-11, 2013

(15) G. X. Ritter and J. N. Wilson : "Computer vision algorithms in image algebra", CRC Press, pp. 184-200, 2000

(16) Y. Hosomi, K. Oyama, T. Honda and T. Ohkawa : “A method of detecting interaction between breeding cows from time-series bird's eye pictures of pasture", IJCNN, International Joint Conference on Neural Networks, pp. 2973-2978, 2016 